\title{
Hallazgos electroencefalográficos en pacientes con diagnóstico confirmado de SARS-CoV-2/COVID-19
}

\section{Electroencephalographic findings in patients with confirmed SARS-CoV-2/COVID-19 diagnosis}

\author{
Paul Shkurovich-Bialik, ${ }^{*}$ Jairo Daniel Evangelio-Miranda, ${ }^{\ddagger}$ Gilberto Ángel Maldonado-Torres*
}

Citar como: Shkurovich-Bialik P, Evangelio-Miranda JD, Maldonado-Torres GÁ. Hallazgos electroencefalográficos en pacientes con diagnóstico confirmado de SARS-CoV-2/COVID-19. An Med (Mex). 2020; 65 (3): 194-200. https://dx.doi.org/10.35366/95676

\section{RESUMEN}

Introducción: La enfermedad por coronavirus 2019 (COVID-19) ha surgido como una pandemia reciente y se caracteriza por un síndrome respiratorio agudo. Recientemente se describen datos de alteraciones en el sistema nervioso central y con expresión clínica que sugiere afección neurológica directa por el virus. Objetivo: Describir los hallazgos de videoelectroencefalografía en pacientes con COVID-19 realizados en el Centro Médico ABC. Material y métodos: Se realizó un análisis retrospectivo de los pacientes diagnosticados con COVID-19 en quienes se practicó videoelectroencefalografía digital. Se recolectó información con respecto a su edad, género, indicación del estudio, gravedad de la enfermedad, estado metabólico, gravedad por imagen tomográfica de tórax, hallazgos de neuroimagen, medicamentos durante el estudio, evolución del paciente y hallazgos electroencefalográficos. Resultados: Se identificaron 14 videoelectroencefalografías en 11 pacientes. Todos los pacientes cuentan con prueba positiva por PCR para COVID-19. Las indicaciones fueron: seis por sospecha de actividad epiléptica, siete por alteración en el estado de alerta y uno por agitación. La edad promedio fue 56 (22-77) con nueve hombres. Por escala de severidad: uno $(9.09 \%)$ cursó con enfermedad leve, uno (9.09\%) con grado moderado, uno (9.09\%) severo y ocho $(72.72 \%)$ con grado crítico. Las videoelectroencefalografías digitales mostraron lentificación de la actividad de fondo, pérdida

\section{ABSTRACT}

Introduction: Coronavirus disease 2019 (COVID-19) has recently emerged as a pandemic characterized by an acute respiratory syndrome. Neurological involvement by the virus have recently been described. To date, there is little evidence of the electroencephalographic (EEG) changes associated with the disease. Objective: To describe the findings of videoelectroencephalography in patients with COVID-19 performed at the ABC Medical Center. Material and methods: $A$ retrospective analysis of electroencephalography of patients diagnosed with COVID-19 was performed. Information regarding age, gender, study indication, disease severity, metabolic status, lung-CT scan severity, neuroimaging findings, medications at the time of the study, patient evolution, and electroencephalographic findings was analyzed. Results: 14 electroencephalography were identified in 11 patients. All patients had SARS-CoV-2/COVID-19 positive PCR test. The indications were: 6 for suspicion of epileptic activity, 7 for alteration in alertness and 1 for agitation. The average age was 56 (22-77), 9 men. By severity scale 1 (9.09\%) had mild illness, 1 (9.09\%) moderate degree, 1 (9.09\%) severe and $8(72.72 \%)$ critical degree. electroencephalography showed background slowing, loss of gradients and poor reactivity in $12(85.71 \%)$ studies, all of them with severe to critically ill disease. Triphasic waves

\footnotetext{
* Especialista en Neurología y Fisiología Clínica.

‡ Especialista en Neurología. Residente en Neurofisiología Clínica.
}

Centro Neurológico, Departamento de Neurofisiología. Centro Médico ABC. México.

Recibido para publicación: 03/05/2020. Aceptado: 12/08/2020

Correspondencia: Paul Shkurovich-Bialik

E-mail: paul.shkurovich@gmail.com

\author{
Abreviaturas: \\ SARS-CoV-2 = Síndrome respiratorio agudo por coronavirus 2. \\ $\mathrm{AEA}=$ Alteración del estado de alerta. \\ SNC $=$ Sistema nervioso central. \\ ECA-2 = Enzima convertidora de angiotensina 2 \\ $\mathrm{EEG}=$ Electroencefalograma \\ VEEG = Videoelectroencefalografía digital .
}


de gradientes y ausencia de reactividad en $12(85.71 \%)$ de los 14 estudios realizados, todos ellos con grado severo a grave de la enfermedad. Se encontraron ondas trifásicas en tres (21.42\%), lentificaciones generalizadas en tres $(21.42 \%)$, patrones periódicos generalizados en dos (14.28\%), uno $(7.14 \%)$ patrón de brote supresión y uno $(7.14 \%)$ con actividad epileptiforme interictal, el cual contaba con antecedente de epilepsia. Conclusión: Los resultados de las videoelectroencefalografías demuestran una correlación entre el estado clínico del paciente con ciertos hallazgos en los registros. Se requieren muestras más grandes y mayor seguimiento de pacientes para confirmar estos hallazgos.

Palabras clave: Electroencefalograma, videoelectroencefalograma, COVID-19, SARS-CoV-2.

Nivel de evidencia: III

\section{INTRODUCCIÓN}

Recientemente la enfermedad por coronavirus 2019 (COVID-19) ha cobrado importancia por sus repercusiones a nivel mundial y fue declarada una pandemia el 11 de marzo de 2020 por la Organización Mundial de la Salud (OMS). ${ }^{1}$ La enfermedad con epicentro inicial en China se ha propagado rápidamente a Europa, Estados Unidos y otros países de América. En la actualidad, la cifra acumulada llega a los 120,102 infectados en México con 14,053 defunciones según datos publicados por el Gobierno de México ${ }^{2}$ hasta el momento de esta publicación.

$\mathrm{El}$ síndrome respiratorio agudo por coronavirus 2 (SARS-CoV-2), que ocasiona la infección COVID-19, habitualmente inicia con manifestaciones respiratorias que pueden progresar rápidamente a condiciones que ponen en riesgo la vida del paciente, particularmente en poblaciones vulnerables. Con el incremento dramático en la incidencia de casos, es claro que las complicaciones de dichos pacientes no se limitan a las vías respiratorias y que, con frecuencia, ocasionan un daño multiorgánico que incluye complicaciones neurológicas. Éstas incluyen cefalea, eventos cerebrovasculares agudos, crisis convulsivas, neuropatías periféricas y diversos grados de alteración del estado de alerta. Esta última cobra particular relevancia, ya que algunos estudios han demostrado que al existir alteración del estado de alerta (AEA) caracterizada por delirio o encefalopatía, especialmente durante las fases iniciales de la infección, la mortalidad es significativamente mayor que en los pacientes que no mostraron AEA. ${ }^{3}$

Las manifestaciones neurológicas en COVID-19 pueden deberse al neurotropismo propio del SARS$\mathrm{CoV}-2$, es decir, representar una manifestación pri- were found in $3(21.42 \%)$, generalized slowing in $3(21.42 \%)$, generalized periodic patterns in 2 (14.28\%), 1 (7.14\%) burstsuppression pattern, and $1(7.14 \%)$ with generalized interictal epileptiform activity in a patient with history of epilepsy. Conclusion: The electroencephalography results correlate between the patient's clinical status with certain findings in the electroencephalography records. Larger samples and longer patient follow-up are required to confirm these findings.

Keywords: Electroencephalogram, video-electroencephalogram, COVID-19, SARS-CoV-2.

Level of evidence: III maria de la infección, o bien ser secundarias a alteraciones metabólicas, hidroelectrolíticas, cardiocirculatorias, inflamatorias, etcétera, pero la incidencia de las complicaciones neurológicas por SARS-CoV-2 se desconoce. ${ }^{4}$ La creciente evidencia indica que los síntomas neurológicos en pacientes con COVID-19 pueden estar relacionados con una infección directa al sistema nervioso central (SNC), ya que existen estudios de otros coronavirus que mostraron neurotropismo y la capacidad de producir encefalitis en seres humanos. ${ }^{5}$ Las hipótesis señalan que el virus puede causar una infección nasal e ingresar al SNC a través del bulbo olfatorio causando inflamación y desmielinización. ${ }^{6}$ Esto apoyado por la prevalencia de disfunción olfativa y gustativa que ha sido reportada en un registro de casos de 12 hospitales europeos, donde 417 pacientes con COVID-19, de leve a moderado, participaron con cuestionarios: $85.6 \%$ y $88 \%$ de los pacientes reportaron trastornos del olfato y del gusto, respectivamente, y la disfunción olfativa fue el síntoma inicial en $12 \%$ de los casos. ${ }^{7}$

La invasión cerebral y el desarrollo de encefalitis clínica se ha reportado por el equipo médico del Hospital Ditan de Beijing, quienes confirmaron la presencia de SARS-CoV-2 en líquido cefalorraquídeo en pacientes con COVID-19. La capacidad de invasión al SNC puede estar relacionada con la afinidad que tiene el virus a la enzima convertidora de angiotensina 2 (ECA-2), la cual también se encuentra expresada en el sistema nervioso. Los datos recientes sugieren afección neurológica con particular expresión en pacientes con COVID-19 severo. ${ }^{8}$ Las neuronas, astrocitos y oligodendrocitos expresan ECA-2 en altas concentraciones en algunas áreas del SNC como: sustancia nigra, ventrículos, giro temporal medial, corteza del cíngulo posterior y el bulbo olfatorio. Otras 
posibles vías de invasión descritas son: transferencia transináptica, infección de endotelio vascular o por migración de leucocitos a través de la barrera hematoencefálica. ${ }^{9}$ De igual forma, algunos estudios reportan la asociación de manifestaciones neurológicas de COVID-19 con hallazgos en imagen encontrando infartos isquémicos, hemorragias, encefalopatía necrosante hemorrágica aguda, leucoencefalopatía y microhemorragias. ${ }^{10}$ Esto apoyando aún más la posible infección al SNC.

La creciente evidencia de infección al SNC y el papel que juega el electroencefalograma (EEG) para valorar las alteraciones del estado de alerta, e incluso algunas infecciones virales del SNC, hacen importante describir patrones que se encuentren en registros de pacientes con COVID-19. De lo poco publicado hasta ahora, se tiene un reporte de caso con ritmo de fondo lento y enlentecimiento focal en región temporal en el EEG de un paciente con COVID-19 y encefalopatía. ${ }^{11}$ Otro reporte de caso describe un EEG con enlentecimiento difuso sin actividad epiléptica en un paciente VIH positivo con encefalopatía que cursó con crisis tónico clónicas. ${ }^{12}$ Finalmente, una serie de casos en la que se describieron los hallazgos de 22 pacientes COVID-19 positivos y se compararon con seis COVID-19 negativos realizados con un montaje simplificado de ocho electrodos, ellos encontraron como principal indicación de EEG la encefalopatía además de describir un incremento en la presencia de descargas epileptiformes principalmente como ondas agudas frontales. ${ }^{13}$ Ante la poca evidencia disponible, es importante reportar todo registro que sume información sobre esta enfermedad y su repercusión al SNC.

El Centro Médico ABC ha mantenido sus operaciones durante la pandemia de COVID-19, y una de sus sedes (campus Observatorio) fue convertido en un centro para la recepción y atención exclusivamente de pacientes con diagnóstico confirmado de COVID-19. Debido a esto, el Departamento de Neurofisiología Clínica realizó los estudios solicitados a pacientes hospitalizados siguiendo las normas institucionales e internacionales para la protección del personal expuesto. Esto ha permitido la realización de un número importante de registros con videoelectroencefalografía digital (VEEG, por sus siglas en inglés) en aquellos pacientes que mostraban alteraciones neurológicas y que tenían indicación para el estudio tras una valoración neurológica.

En este trabajo presentamos los primeros hallazgos de VEEG en pacientes ingresados a la Unidad de Cuidados Intensivos (UCI) que se obtuvieron en nuestro centro.

\section{MATERIAL Y MÉTODOS}

Se realizó un análisis ambispectivo preliminar de los registros de VEEG en pacientes con diagnóstico de COVID-19 dentro del Centro Médico ABC Campus Observatorio confirmados por PCR mediante muestreo con hisopado faríngeo. Las solicitudes de VEEG fueron realizadas por un neurólogo que inicialmente valoró al paciente desde el punto de vista clínico y sólo fueron realizados aquellos estudios en donde se verificó la naturaleza urgente del mismo.

Se limitó a sólo un técnico para la realización del trazo para evitar mayor exposición de personal. Éste fue capacitado y contaba con todas las medidas indicadas en protocolos de protección y prevención de infecciones, implementadas por la institución y publicadas en guías actuales. ${ }^{14,15}$ Los estudios fueron practicados mediante un equipo de VEEG digital marca Cadwell modelo Easy II EEG System y siguiendo las recomendaciones de la Federación Internacional de Neurofisiología Clínica utilizando el Sistema Internacional 10/20. Se realizó la interpretación por el personal del Departamento de Neurofisiología Clínica, todos ellos avalados por el Consejo Mexicano de Neurofisiología Clínica.

Se recolectaron datos del historial médico con respecto a: edad, género, antecedentes de enfermedad neurológica, alteraciones metabólicas y tratamiento al momento del estudio, hallazgos de neuroimagen (tomografía o resonancia magnética) y desenlace de la evolución del paciente. Se determinó la severidad de la enfermedad por la clasificación Criteria for classification of COVID-19 severity, ${ }^{16}$ publicada recientemente y mediante imagen por tomografía de tórax de acuerdo con la propuesta del Instituto Nacional de Enfermedades Respiratorias de México. ${ }^{17-20}$ Se identificó por expediente clínico la indicación para el VEEG y se clasificó en tres grupos: agitación o psicosis, alteración del estado de alerta y sospecha de actividad epiléptica.

Los estudios de VEEG fueron interpretados por dos neurofisiólogos de manera independiente y se obtuvieron datos con respecto a: número de días desde el inicio de síntomas por COVID-19 a la realización del estudio, duración del registro, actividad de fondo, presencia de gradientes de frecuencias y voltajes, presencia de reactividad a la apertura ocular y estimulación sensorial, identificación de lentificación, patrones específicos, patrones periódicos y actividad epileptiforme.

\section{RESULTADOS}

Se identificaron 14 VEEG en 11 pacientes dentro del periodo del 22 de abril al 09 de junio de 2020, 
con una duración promedio de 34.9 minutos y un rango de 24-45 minutos, además de un promedio de 32.7 (14-51) días desde el inicio de síntomas por COVID-19 a la realización del estudio. Todos los pacientes cuentan con prueba positiva por PCR para COVID-19. Las indicaciones para los estudios fueron: seis por sospecha de actividad epiléptica, siete por alteración en el estado de alerta y uno por agitación.

La edad promedio de los pacientes de la muestra $(\mathrm{n}=11)$ fue $56(22-77)$ con nueve hombres. De éstos, 10 (90.9\%) no tenían antecedente de enfermedad neurológica y sólo uno (9.09\%) tenía antecedente de epilepsia. Durante los estudios de VEEG se encontró que cinco $(45.45 \%)$ cursaban con insuficiencia renal, uno $(9.09 \%)$ con trastorno hidroelectrolítico (hipernatrémico) y cinco $(45.45 \%)$ sin alteraciones metabólicas. Al agruparlos por escala de severidad, uno $(9.09 \%)$ cursó con una enfermedad leve, uno $(9.09 \%)$ con grado moderado, uno $(9.09 \%)$ severo y ocho $(72.72 \%)$ con grado crítico. Cuatro pacientes fallecieron, todos en estado crítico (50\% del total de pacientes críticos) y sólo siete (50\% pacientes críticos y $100 \%$ de los no críticos) sobreviven al momento de esta publicación. Los datos demográficos, así como la clasificación por escala de severidad de acuerdo con la imagen tomográfica de tórax y los hallazgos de neuroimagen (tomografía o resonancia magnética de cráneo) se describen en las Tablas 1 y 2.

Los registros de VEEG mostraron lentificación de la actividad de fondo, pérdida de gradientes y ausencia de reactividad en $12(85.71 \%)$ de los 14 estudios realizados, en todos los pacientes graves y críticos. Se encontraron ondas trifásicas en tres (21.42\%) estudios, lentificaciones generalizadas en tres $(21.42 \%)$, patrones periódicos generalizados en dos (14.28\%) y un $(7.14 \%)$ patrón de brote supresión (paciente sedado con dexmedetomidina, midazolam y fentanilo), todo esto en pacientes en estado crítico. Por último, sólo un registro mostró actividad epileptiforme generalizada en un paciente con enfermedad moderada y con el antecedente de epilepsia. La descripción de los hallazgos, así como su relación con cada paciente se encuentra en la Figura 1.

\section{DISCUSIÓN}

En nuestro estudio la principal causa para solicitud de VEEG en pacientes positivos a COVID-19 fue la AEA, estrechamente relacionado con estados encefalopáticos, en segundo lugar la sospecha de actividad epiléptica y por último la agitación. Esto fue similar en los tres estudios mencionados con reportes de EEG, en los que la principal indicación fue un estado encefalopático con AEA. ${ }^{6-8}$ Encontramos en todos los pacientes con grado severo y crítico de la enfermedad un enlentecimiento de la actividad de fondo asociado con pérdida de gradientes y ausencia de reactividad. Esto no se presentó en aquellos pacientes con grados moderados y leves de la enfermedad, lo que sugiere un probable correlato entre los hallazgos de VEEG con la expresión clínica de COVID-19. Esto fue similar a lo descrito por Gala-

Tabla 1: Datos demográficos, severidad, hallazgos de electroencefalograma (EEG) y evolución de pacientes.

Cada paciente se representa por un número del 1 al 11 con sus respectiva información.

\begin{tabular}{|c|c|c|c|c|c|c|c|c|c|}
\hline 1 & 77 & $M$ & Ninguno & Crítico & Severo & $\mathrm{Mh}$ & IR & LIG & Finado \\
\hline 2 & 74 & $M$ & Ninguno & Crítico & Severo & $\mathrm{Mh}$ & IR & OT & Finado \\
\hline 3 & 49 & $M$ & Epilepsia & Moderado & Leve & Normal & Ninguno & DEI & Vivo \\
\hline 4 & 60 & $M$ & Ninguno & Crítico & Severo & Normal & Ninguno & LIG & Vivo \\
\hline 7 & 70 & $M$ & Ninguno & Crítico & Severo & Normal & Ninguno & Lento & Vivo \\
\hline 8 & 25 & $F$ & Ninguno & Leve & Normal & Normal & Ninguno & Normal & Vivo \\
\hline 9 & 30 & $M$ & Ninguno & Crítico & Severo & $\mathrm{H}, \mathrm{LD}$ y $\mathrm{Mh}$ & $\mathrm{TH}$ & LIG & Finado \\
\hline 10 & 64 & $M$ & Ninguno & Crítico & Severo & $N / A$ & Ninguno & OT & Vivo \\
\hline 11 & 45 & $M$ & Ninguno & Crítico & Severo & $\mathrm{Mh}$ & IR & BS & Vivo \\
\hline
\end{tabular}

$M=$ Masculino, $F=$ Femenino, Mh = Microhemorragias, $I=$ Isquémico, $\mathrm{H}=$ Hemorragia, $L D=$ Leucoencefalopatía Difusa, IR = Insuficiencia Renal, $T H=$ Trastorno Hidroelectrolítico, LIG = Lentificación Intermitente Generalizada, OT = Ondas Trifásicas, DEI = Descargas Epilépticas Interictales, BS = Brote Supresión. 
Tabla 2: Hallazgos VEEG de pacientes con COVID-19.

\begin{tabular}{|c|c|c|c|c|c|c|c|c|c|c|c|c|}
\hline $\begin{array}{l}\mathrm{N} \text { de paciente } \\
\text { Fecha de } \\
\text { electroence- } \\
\text { falograma }\end{array}$ & Indicación & $\begin{array}{l}\text { Actividad } \\
\text { de fondo }\end{array}$ & Gradiente & $\begin{array}{l}\text { Reactivi- } \\
\text { dad }\end{array}$ & $\begin{array}{l}\text { Lentifica- } \\
\text { ción }\end{array}$ & $\begin{array}{c}\text { Patrón } \\
\text { Específico }\end{array}$ & $\begin{array}{l}\text { Patrón } \\
\text { Periódico }\end{array}$ & $\begin{array}{l}\text { Actividad } \\
\text { Epiléptica }\end{array}$ & $\begin{array}{l}\text { Clasifica- } \\
\text { ción de } \\
\text { gravedad }\end{array}$ & $\begin{array}{l}\text { Estado } \\
\text { metabó- } \\
\text { lico }\end{array}$ & Fármaco & $\begin{array}{l}\text { Desen- } \\
\text { lace }\end{array}$ \\
\hline $\begin{array}{c}(1) \\
22 / 04 / 20\end{array}$ & SAE & Lenta & Perdido & Ausente & $I G$ & Ninguno & Ninguno & Ninguno & Crítico & IR & $S$ & $\mathrm{~F}$ \\
\hline $\begin{array}{c}(2) \\
26 / 04 / 20\end{array}$ & AEA & Lenta & Perdido & Ausente & Ninguna & Ninguno & Ninguno & Ninguno & Crítico & IR & $S$ & $\mathrm{~F}$ \\
\hline $03 / 05 / 20$ & AEA & Lenta & Perdido & Ausente & Ninguna & ОТ & Ninguno & Ninguno & Crítico & $\mathrm{IR}$ & Ninguno & $\mathrm{F}$ \\
\hline $\begin{array}{c}(3) \\
05 / 05 / 20\end{array}$ & AEA & Normal & Normal & Presente & Ninguno & Ninguno & $\mathrm{G}$ & $\mathrm{G}$ & Moderado & Ninguno & FAE & V \\
\hline $\begin{array}{c}(4) \\
04 / 05 / 20\end{array}$ & A & Lenta & Perdido & Ausente & IG & Ninguno & Ninguno & Ninguno & Crítico & Ninguno & $S$ & V \\
\hline $\begin{array}{c}(5) \\
10 / 05 / 20\end{array}$ & AEA & Lenta & Perdido & Ausente & Ninguno & Ninguno & Ninguno & Ninguno & Crítico & IR & $S$ & $\mathrm{~F}$ \\
\hline $\begin{array}{c}(7) \\
21 / 05 / 20\end{array}$ & SAE & Lenta & Perdido & Ausente & Ninguna & Ninguno & Ninguno & Ninguno & Crítico & Ninguno & S & V \\
\hline $\begin{array}{c}(8) \\
22 / 05 / 20\end{array}$ & SAE & Normal & Normal & Presente & Ninguna & Ninguno & Ninguno & Ninguno & Leve & Ninguno & Ninguno & V \\
\hline $\begin{array}{c}(9) \\
29 / 05 / 20\end{array}$ & SAE & Lenta & Perdido & Ausente & IG & Ninguno & Ninguno & Ninguno & Crítico & $\mathrm{TH}$ & $\mathrm{FAE}+\mathrm{S}$ & $\mathrm{F}$ \\
\hline $\begin{array}{c}(10) \\
04 / 06 / 20\end{array}$ & AEA & Lenta & Perdido & Ausente & Ninguna & ОТ & $G$ & Ninguno & Crítico & Ninguno & $S$ & V \\
\hline $\begin{array}{c}(11) \\
09 / 06 / 20\end{array}$ & SAE & Lenta & Perdido & Ausente & Ninguna & BS & Ninguno & Ninguno & Crítico & IR & $S$ & V \\
\hline
\end{tabular}

nopoulou AS y colegas ${ }^{13}$ que reportan, en su serie de 22 pacientes COVID-19 positivos, un enlentecimiento de fondo y pérdida de gradientes, la mayoría de ellos con ventilación mecánica y, por lo tanto, con un grado crítico de la enfermedad.

Es importante mencionar en nuestro estudio la presencia de ondas trifásicas y enlentecimientos intermitentes generalizados, los cuales se presentaron únicamente en pacientes con estado crítico donde la mayoría evolucionaban a un desenlace fatal y aquéllos que sobreviven continúan hospitalizados y graves. Estos hallazgos no se reportaron en ningún otro estudio previo que haya estudiado con EEG a pacientes con COVID-19.

Galanopoulou AS y colaboradores ${ }^{13}$ reportan en su estudio el incremento de actividad epileptiforme de predominio frontal en nueve de sus pacientes, algo que no encontramos en ninguno de nuestros registros. La única actividad epiléptica registrada en nuestro estudio fue generalizada en un paciente con antecedente de epilepsia. También encontramos un registro que mostraba actividad de brote supresión, probablemente relacionado al uso de sedantes durante el estudio (midazolam, dexmedetomidina y propofol).

Los reportes en imagen, si bien concuerdan con lo reportado hasta ahora en la literatura como microhemorragias, leucoencefalopatía difusa, eventos vasculares hemorrágicos e isquémicos, no tuvieron una correlación importante con lo encontrado en estudios de VEEG.

Los cambios que observamos en nuestro estudio con VEEG pueden ser explicados por causas secundarias como alteraciones metabólicas, hidroelectrolíticas, inflamatorias, cardiocirculatorias o por la afección pulmonar severa, pero también pudiesen ser una manifestación directa de infección primaria al sistema nervioso central y que estos patrones de ondas trifásicas y enlentecimientos generalizados puedan ser una expresión directa de infección, tomando en cuenta que algunos pacientes no tenían ninguna alteración 


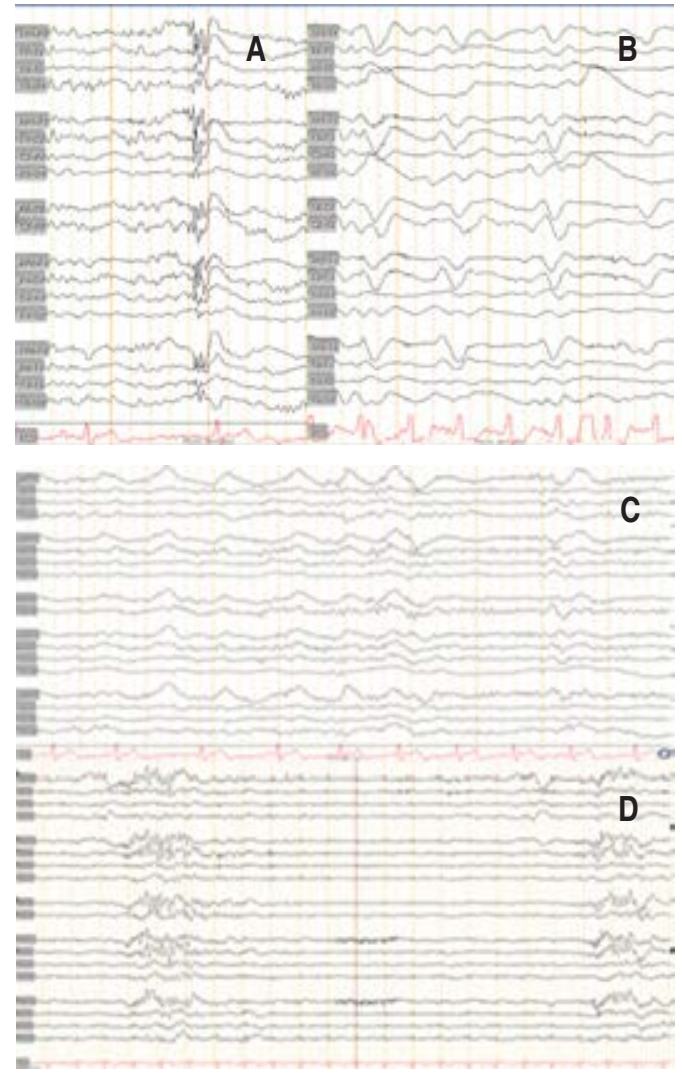

Figura 1: Electroencefalogramas. (A) con actividad epileptiforme y (B) con ondas trifásicas. Paciente 3 y 4 , respectivamente; tomado con sensibilidad de 5 microvoltios y velocidad de 10 segundos (C) con actividad periódica generalizada y (D) con patrón de brote supresión. Paciente 3 y 11 , respectivamente, el primero tomado con sensibilidad de 5 microvoltios y velocidad de 10 segundos y el segundo con sensibilidad de 2 microvoltios y velocidad de 24 segundos.

metabólica en el momento del estudio. Se requiere de una muestra más amplia, además de complementar con estudios que permitan confirmar la presencia del virus en el SNC, como sería la toma de líquido cefalorraquídeo con prueba de PCR como lo hizo el equipo médico en el Hospital de Ditan de Beijing ${ }^{8}$ o bien, realizando biopsias a los cerebros de pacientes enfermos nuevamente para aislar el patógeno.

Nuestro estudio es pionero en reportar cambios en VEEG en pacientes con COVID-19 con la ventaja de realizarse implementando un montaje completo de 21 electrodos y el sistema 10-20. Todos ellos fueron realizados por técnicos con formación académica previa e interpretados por neurofisiólogos certificados. Otra gran ventaja fue la accesibilidad para la información de la evolución clínica de estos pacientes, además de confirmación de todos ellos mediante pruebas de PCR.
Dentro de nuestras limitaciones es importante mencionar el numero pequeño de la muestra, que si bien, el Centro Médico ABC Campus Observatorio cuenta con varios pacientes con diagnóstico de COVID-19, no todos cumplen los criterios e indicaciones para la realización de estudios de VEEG, procurando así a aquellos de suma importancia para la decisión terapéutica del paciente y limitando la exposición de nuestro personal con estudios innecesarios.

A pesar de estas limitaciones, creemos que la alta incidencia de manifestaciones neurológicas de pacientes con COVID-19, particularmente aquéllos que cursan con un cuadro grave, podría demostrar en series más grandes y de mayor tiempo de seguimiento, subpoblaciones de pacientes que podrían tener un comportamiento predecible basado en los hallazgos electroencefalográficos, especialmente al tratarse de una prueba rápida y sencilla que puede realizarse dentro de la Unidad de Cuidados Intensivos, y que al seguir las recomendaciones ya establecidas, pueden practicarse de manera segura y objetiva sin exponer de manera inapropiada al personal de salud.

\section{CONCLUSIÓN}

Nuestro estudio demuestra una clara correlación entre el VEEG y la evolución clínico-radiológica del paciente con enfermedad respiratoria causada por el virus SARS-CoV-2 y su expresión como COVID-19, y sugiere ser de gran utilidad en la evaluación integral del paciente que cursa con manifestaciones neurológicas. Además, se describen la presencia de patrones no descritos previamente que pudiesen tener una muy estrecha relación con grados más severos de la enfermedad y, quizá, con datos sugestivos de infección al SNC. Se requieren más estudios que confirmen estas teorías y el análisis de un mayor número de pacientes.

\section{BIBLIOGRAFÍA}

1. Ahmad I, Rathore FA. Neurological manifestations and complications of COVID-19: A literature review. J Clin Neurosci. 2020; 77: 8-12.

2. Coronavirus [Internet]. gob.mx. [cited 2020 Jun 9]. Available from: https://coronavirus.gob.mx

3. Mao L, Wang M, Chen S, He Q, Chang J, Hong C et al. Neurologic manifestations of hospitalized patients with coronavirus disease 2019 in Wuhan, China. JAMA Neurol. 2020; 77 (6): 1-9.

4. Zhou L, Zhang M, Wang J, Gao J. SARS-CoV-2: underestimated damage to nervous system. Travel Med Infect Dis. 2020; 101642.

5. Natoli S, Oliveira V, Calabresi P, Maia LF, Pisani A. Does SARS-Cov-2 invade the brain? Translational lessons from animal models. Eur J Neurol. 2020; 10.1111/ene.14277. 
An Med (Mex) 2020; 65 (3): 194-200

6. Asadi-Pooya AA, Simani L. Central nervous system manifestations of COVID-19: A systematic review. J Neurol Sci [Internet]. 2020 [cited 2020 Apr 25]; 413: 116832. Available from: https://www.jns-journal.com/article/S0022$510 X(20) 30168-4 / p d f$

7. Lechien JR, Chiesa-Estomba CM, De Siati DR, Horoi M, Le Bon SD, Rodriguez A et al. Olfactory and gustatory dysfunctions as a clinical presentation of mild-to-moderate forms of the coronavirus disease (COVID-19): a multicenter European study. Eur Arch Otorhinolaryngol. 2020; 277 (8): 2251-2261.

8. Lahiri D, Ardila A. COVID-19 pandemic: a neurological perspective. Cureus. 2020; 12 (4): e7889.

9. Zubair AS, McAlpine LS, Gardin T, Farhadian S, Kuruvilla DE, Spudich S. Neuropathogenesis and neurologic manifestations of the coronaviruses in the age of coronavirus disease 2019: a review. JAMA Neurol. 2020; 10.1001/jamaneurol.2020.2065.

10. Radmanesh A, Derman A, Lui YW, Raz E, Loh JP, Hagiwara M et al. COVID-19 -associated diffuse leukoencephalopathy and microhemorrhages. Radiology. 2020; 202040.

11. Filatov A, Sharma P, Hindi F, Espinosa PS. Neurological complications of coronavirus disease (COVID-19): encephalopathy. Cureus. 2020; 12 (3): e7352.

12. Haddad S, Tayyar R, Risch L, Churchill G, Fares E, Choe M et al. Encephalopathy and seizure activity in a COVID-19 well controlled HIV patient. IDCases. 2020; 21: e00814.

13. Galanopoulou AS, Ferastraoaru V, Correa DJ et al. EEG findings in acutely ill patients investigated for SARS-CoV-2/
COVID-19: A small case series preliminary report. Epilepsia Open. 2020; 5 (2): 314-324.

14. Guidance for clinical neurophysiology examination throughout the COVID-19 pandemic. Latin American chapter of the IFCN task force - COVID-19. Clin Neurophysiol. 2020; 131 (7): 1589-1598.

15. Gélisse P, Rossetti AO, Genton P, Crespel A, Kaplan PW. How to carry out and interpret EEG recordings in COVID-19 patients in ICU? Clin Neurophysiol. 2020; 131 (8): 2023-2031.

16. Lu L, Xiong W, Liu D, Liu J, Yang D, Li N et al. New onset acute symptomatic seizure and risk factors in coronavirus disease 2019: a retrospective multicenter study. Epilepsia. 2020; 61 (6): e49-e53.

17. COVID-19 Diagnóstico por imagen y espectro radiológico [Internet]. gob.mx. [cited 2020 Jun 9]. Available from: https:// www.gob.mx/salud/iner/videos/covid-19-diagnostico-porimagen-y-espectro-radiologico?idiom $=\mathrm{es}$

18. Yuan M, Yin W, Tao Z, Tan W, Hu Y. Association of radiologic findings with mortality of patients infected with 2019 novel coronavirus in Wuhan, China. PLoS One. 2020; 15 (3): e0230548.

19. Wang Y, Dong C, Hu Y, Li C, Ren Q, Zhang X et al. Temporal changes of CT findings in 90 patients with COVID-19 pneumonia: a longitudinal study. Radiology. 2020; 296 (2): E55-E64.

20. Pan F, Ye T, Sun P, Gui S, Liang B, Li L et al. Time course of lung changes at chest CT during recovery from coronavirus disease 2019 (COVID-19). Radiology. 2020; 295 (3): 715-721. 